\title{
Les migrations au musée ! Sciences sociales et muséographie sont-elles complémentaires?
}

Migrations in Museums! Are Social Sciences and Museography Complementary? ¡La migración en los museos! ¿Son complementarias las ciencias sociales y la museografía?

\section{Yann Scioldo-Zürcher}

\section{CpenEdition}

Journals

\section{Édition électronique}

URL : https://journals.openedition.org/remi/8236

DOI : $10.4000 /$ remi.8236

ISSN : $1777-5418$

Éditeur

Université de Poitiers

Édition imprimée

Date de publication : 1 décembre 2016

Pagination : 163-183

ISBN : 979-10-90426-29-0

ISSN : 0765-0752

\section{Référence électronique}

Yann Scioldo-Zürcher, « Les migrations au musée ! Sciences sociales et muséographie sont-elles complémentaires ? », Revue européenne des migrations internationales [En ligne], vol. 32 - n³ et 4 | 2016, mis en ligne le 01 décembre 2018, consulté le 14 avril 2022. URL : http:// journals.openedition.org/remi/8236; DOI : https://doi.org/10.4000/remi.8236 


\section{Les migrations au musée! Sciences sociales et muséographie sont-elles complémentaires?}

\section{Yann Scioldo-Zürcher ${ }^{1}$}

Les musées de société entièrement ou partiellement consacrés aux faits migratoires ont connu un important développement en Europe, à partir du milieu des années 1990. De nouveaux établissements d'envergure nationale, régionale et locale ont ainsi ouvert leurs portes, venant alors par leurs parcours permanents, pérenniser le succès d'expositions temporaires (Poli et IdjéraouiRavez, 2011 ; Meza-Torres, 2011). Et tous réaffirment dans un contexte de montée des mouvements xénophobes, combien les migrations sont partie intégrante des histoires de chaque pays. Cependant, ces rétrospectives sont souvent devenues " prisonnières d'un nationalisme muséographique [...], [tant elles ont été] élaborées à l'aune de la construction du récit national, [et] inscrites dans la perspective de l'intégration " (Grosfoguel et al., $2011: 7$ ) ; et de fait, elles ont tendance à minimiser les mobilités transnationales et I'histoire complexe des circulations. Dans le cadre de ce numéro anniversaire de la REMI, il nous a ainsi paru intéressant " d'observer " quelques muséums de renommée internationale (à Paris, Hambourg, Berlin, Tel Aviv et Moscou) consacrés à I'histoire des migrations et des sociétés juives en diaspora, à un moment où les connaissances, en termes de narrations événementielles et de méthodologies conceptuelles, étaient suffisamment " opérantes " pour exposer les faits dans leur complexité2.

1 Historien, Chargé de recherche au CNRS, Centre de recherche français à Jérusalem (UMIFRE 7, CNRS-MAEDI), 3 Shimshon Street, Baka, P.B. 547, 91004 Jérusalem, Israël ; yann.scioldozurcher@cnrs.fr

2 Nous avons ainsi, selon un choix non exhaustif, visité au cours du mois de novembre 2015 des musées spécifiquement consacrés aux faits migratoires : le Musée de l'émigration Ballinstadt de Hambourg et le Musée de I'histoire de l'immigration de Paris. Pour illustrer la question des représentations muséales des diasporas, nous avons aussi porté notre étude sur les musées d'histoire juive : Musée d'art et d'histoire du Judaïsme de Paris, Musée juif et Centre de la tolérance de Moscou, Musée juif de Berlin et Musée de la diaspora (Beit Hatefutsoth) de Ramat-Aviv (situé sur le campus de l'Université de Tel-Aviv). Aux visites des expositions permanentes, a été jointe, pour cet article, l'étude des guides d'exposition. Respectivement : pour le Musée Ballinstadt : Ballinstadt, The Emigration Museum (sans date) ; pour le Musée de I'histoire de l'immigration : Guide de I'exposition permanente (2009) et Guide de la Galerie des dons (2014) ; pour le Musée d'art et d'histoire du Judaïsme : Guide des collections (1999) ; pour le Musée juif et le Centre de la tolérance de Moscou : Atlas of Russian Jewish History based on Jewish Museum and Tolerance centre Materials (2014) ; pour le Musée juif de Berlin : Musée juif de Berlin. Les essentiels (2010); pour le Musée de la diaspora : Beit Hatfutsot (sans date). Les citations tirées des ouvrages en anglais sont de notre traduction. 
Les savoirs scientifiques ont-ils suffisamment pu peser sur les représentations muséographiques ? Quelles sont les " conventions d'écriture " lorsque les contraintes narratives des directeurs et conservateurs de musées vis-à-vis de leurs tutelles peuvent être en opposition avec les exigences des chercheurs, et lorsque les muséographes ont, par souci de vulgarisation, tendance à vouloir privilégier une approche narrative et linéaire des migrations ? Que nous donnent à penser les parcours permanents, lorsqu'ils sont à la fois des expositions de points de vue, de savoirs et des émotions et que les " tensions entre représentations sont toujours multiples, entre histoire et art, entre texte et image " (Green, 2011 : 135) dans un environnement muséal qui se situe au carrefour du temps historique et du présent?

\section{Des migrants et des minorités juives en musées}

Les musées visités ont tous bénéficié de financements conséquents. Ils ont regroupé dans leurs comités scientifiques chercheurs, équipes de l'établissement (conservateurs, iconographes, anthropologues, historiens d'art, etc.) et muséographes pour proposer de "belles " expositions. Au sens où la muséographie est élaborée et donne à voir œuvres d'art, documents d'archives et iconographiques parfois inédits.

\section{Les musées d'histoire des migrations à Hambourg et à Paris}

Le Musée Ballinstatd de Hambourg, qui a ouvert ses portes en 2007, est situé à l'emplacement d'un ancien " hall d'émigration " par lequel ont transité, entre 1901 et 1938, la plupart des migrants originaires d'Europe centrale et orientale en partance pour le continent américain. Construit sur la petite île de Veddel, sur les rives de l'Elbe, il était financé par la compagnie maritime HAPAG et la municipalité de Hambourg. La première craignait de devoir prendre financièrement à sa charge le voyage-retour de ceux non admis à pénétrer aux États-Unis et les sélectionnait là, avec l'aide des agents de la douane américaine. La seconde, redoutant la criminalité et les épidémies, préférait cantonner les voyageurs à la périphérie de la ville, à proximité de leurs lieux d'embarquement. Le centre avait ainsi pour vocation d'accueillir les migrants, de les héberger et de faciliter leur embarquement depuis un quai judicieusement nommé Amérique. Fermé en 1938, après la Nuit de cristal, devenu par la suite camp de prisonniers, centre de relogement, restaurant et lieu alternatif, il fut pratiquement détruit. Seuls trois bâtiments ont subsisté sur un ensemble qui en comptait trente (dont une église, des réfectoires, des dortoirs, des salles de bain, une centrale électrique, etc.). Le musée, qui présente une histoire de l'émigration depuis Hambourg entre 1850 et 1938, propose ainsi un parcours d'exposition en forte résonnance avec les lieux. La muséographie conte les quatre étapes connues par les migrants d'alors (et admis à poursuivre leur voyage) : une première section retrace l'arrivée à Veddel ; une seconde le déroulement de la vie dans le centre de transit (un dortoir est notamment présenté). Le parcours muséal conduit ensuite le visiteur vers une reconstitution, en miniature, d'un bateau baptisé " des rêves ". Après avoir traversé un quai symbolique, il entre dans la coque du navire, où sont présentées les conditions d'un voyage en soute vers 1870 , et une cabine de seconde classe à la veille de la Première Guerre mondiale. Le visiteur y ressent les risques encourus par les migrants d'alors et apprend la tragédie 
du Cimbria, qui a sombré en 1883 , ne laissant que cinquante-cinq survivants sur 620 embarqués. Enfin, le dernier corps de bâtiment, et quatrième temps de l'exposition, est consacré à l'arrivée (aux États-Unis ou en Amérique du Sud). Les épreuves du passage de la frontière à Ellis Island sont mises en scène et l'exposition se clôt sur la vie quotidienne dans le pays d'installation, notamment à New York, en insistant sur la poursuite d'une vie dans sa communauté et religion d'origine ("Little-Germany ", Brookline). Enfin, en marge de son exposition permanente, le musée propose une section généalogique, où les visiteurs peuvent interroger une base de données composée des noms des migrants qui ont transité en ces lieux.

La convention d'écriture est ici simple : le musée raconte I'histoire d'une trajectoire, du départ du domicile à l'arrivée dans le " nouveau monde ", en ayant recours à une muséographie chargée en émotions. On ne trouve pas de beaux-arts dans l'exposition, mais des valises, de très nombreux documents, archives et photographies, des recréations d'ambiance (le "bateaux des rêves ", le bureau d'Albert Ballin, directeur de la Compagnie, une rue new yorkaise, une autre très folklorisée d'Amérique latine). Quelques témoignages sont enregistrés, audibles en allemand et en anglais. Des "films d'époque " sont aussi diffusés. La conception muséographique très narrative ne s'étend guère du point de vue scientifique, sur des sentiers historiques critiques. On découvre une histoire migratoire linéaire, sans grandes aspérités, qui se termine sans surprise, au terme d'un parcours en étapes, par une évocation de l'intégration dans un nouveau pays. Très symboliquement, le livret de l'exposition reproduit, dans ses dernières pages, un certificat américain de citoyenneté (Ballinstadt Museum, sans date : 58).

Enfin, de façon très complémentaire avec le sentiment d'empathie que l'exposition suscite, le musée délivre à l'attention du visiteur un message politique explicite : I'Allemagne, avant d'être devenue un pays d'immigration, a vu sa population partir, dans des situations parfois complexes, sinon dramatiques (le musée évoque I'histoire de Juifs allemands contraints à la fuite). II en découle ainsi un "devoir de mémoire ", en lien avec les " devoirs citoyens " du temps présent. Le chapitre du livret " faire directement l'expérience de l'émigration " (Ballinstadt Museum, sans date : 56), décrit ainsi I'actuel quartier de Veddel et les actions sociales qui y sont menées en faveur des migrants. En insistant sur le caractère " multiculturel " du quartier $(60 \%$ des habitants de l'île sont d'origine étrangère, $15 \%$ des enfants de l'école primaire de la ville sont allemands de naissance), il rappelle que les immigrés ont aussi légitimité à s'installer définitivement. En illustre le témoignage d'un homme turc, vendeur retraité de vêtements et père de six enfants élevés en Allemagne : " J'ai toujours voulu retourner en Turquie pour ma retraite mais je ne peux pas le faire [...]. Quatre de mes enfants sont déjà mariés, j'ai neuf petits-enfants. Je ne peux pas partir maintenant [...], je ne me sentirais pas plus à la maison dans mon village turc qu'ici. Je connais tous mes voisins et tous me connaissent [...] ". S'ensuit enfin le trombinoscope " multiethnique " des élèves de l'école élémentaire du quartier: "L'émigration fait partie de la vie de tous les jours sur l'île de Veddel, qui en témoigneraient mieux que les élèves de l'école de Slomanstieg ? (Ballinstadt Museum, sans date : 57 ). 
Le Musée de I'histoire de l'immigration de Paris propose, quant à lui, une exposition permanente beaucoup plus ambitieuse en termes scientifiques ${ }^{3}$. Son positionnement sur la scène muséographique nationale et internationale a cependant souffert de plusieurs embarras qui ont fortement nui à sa visibilité ${ }^{4}$. Le choix du lieu fut tout d'abord polémique. L'ancien et imposant Palais des colonies, construit Porte dorée à I'occasion de I'Exposition coloniale de 1931, devenu au terme des indépendances Musée des Arts africains et océaniens, et vidé de ses collections après l'ouverture du Musée du Quai Branly, fut pragmatiquement choisi comme il avait l'avantage d'offrir un " espace disponible " au cœur de la capitale. Mais cela brouilla d'emblée le message du musée, en supposant implicitement que les seuls migrants venus en France étaient originaires des territoires anciennement coloniaux. Une seconde tension fut mise au jour au moment de son ouverture. L'élection de Nicolas Sarkozy à la présidence de la République quelques semaines auparavant et la création d'un ministère de I'Immigration et de I'Identité nationale qui s'ensuivit par le gouvernement François Fillon, avaient entraîné la démission de la presque totalité des historiens du comité scientifique qui dénonçaient alors l'instrumentalisation des immigrés pour des objectifs électoraux et l'opposition ainsi créée entre immigration et identité nationale (Poli et Idjéraoui-Ravez, 2011 : 139). Et le nouveau président n'inaugura pas le musée. Ce fut chose faite sept années plus tard, par François Hollande, son successeur.

Au-delà de ces difficultés liées aux débats sur l'immigration en France, l'exposition permanente s'avère confuse. Elle est d'ailleurs, chose rare, critiquée par la direction même de l'établissement ; son directeur explique son manque de pédagogie par la précipitation à l'ouvrir (Gruson, 2011 : 14). Le pari du musée était ambitieux, trop sans doute. Il avait été décidé de ne pas présenter I'histoire de deux siècles d'immigration en France selon un ordre chronologique, mais thématique et d'illustrer les faits du passé de façon exhaustive. L'exposition permanente se décline ainsi en dix sections reléguant la narration historique à un second plan (sur des tables dites " repères ") : "Émigrer ", "Face à l'État ", "Terre d'accueil, France hostile ", " Ici et là-bas ", "Lieux de vie ", "Au travail ", "Enracinements ", "Sport ", "Religions ", "Culture " scandent le parcours du visiteur tout en gommant les processus qui ont progressivement fait de la France un des pays d'immigration les plus importants au monde. L'exposition propose ainsi de très nombreuses archives, tout en ayant recours à la médiation de l'art, dont l'art contemporain. Et les œuvres présentées tout au long du parcours peuvent parfois plus donner l'impression de brouiller le message qu'illustrer

3 Auparavant dénommée Cité nationale de I'histoire de l'immigration. La Cité est devenue Musée en 2012.

4 L'auteur de l'article a travaillé pendant quelques mois dans le musée, entre 2006 et 2007. Alors jeune docteur, il était en charge de construire l'exposition permanente, en lien avec les instances du musée et le comité scientifique. 
les faits migratoires ${ }^{5}$. En effet, ces dernières ont pour vocation, selon Jacques Toubon $^{6}$, président du Conseil d'orientation, de mettre en relation " récit et émotion ", parfois " en opposition avec le récit des historiens " (Renart et Poinsot, $2011: 25$ et 23 ). II en ressort une exposition permanente confondant histoire de l'immigration et traitement de l'immigration en art, musée traditionnel et musée de société. Cela fut le résultat de la confrontation entre une direction de musée très attachée aux beaux-arts et un comité scientifique soulagé de voir évoquer des exemples historiques essentiels à ses yeux, mais noyés dans un ensemble trop dense ${ }^{7}$. Pour pallier le manque de clarté historique, une chronologie a ainsi dû être rajoutée, mais dans les escaliers menant à l'exposition. Et la difficulté de lecture dans cet espace exigu ne résout pas le problème. Le livret édité, quant à lui, réinjecte la chronologie manquante (Cité nationale de l'histoire de l'immigration, 2009 : 14-15) et offre la possibilité de s'imprégner des documents d'archives présentés dans l'exposition, trop nombreux pour être lus en détail au cours d'une visite classique. Un film retraçant deux siècles d'immigration en France, disponible sur le site internet du musée, complète une exposition manquant de didactique.

Par ailleurs, le message politique porté par l'institution n'est pas clair. La "Cité " se voulait en lien avec la société française ; son conseil d'orientation réunissait jusqu'en 2012 une majorité de membres issus " de la société civile " (Gruson, 2011 : 16). Mais le délitement associatif et le contexte difficile de son ouverture ont brouillé le message d'un établissement qui souhaitait rendre visible " un patrimoine de l'immigration commun à une majorité de Français ". Et contrairement au musée de Hambourg, le livret de l'exposition ne fait jamais mention de thématiques portant sur la politique contemporaine d'immigration ou l'engagement citoyen à connaître cette histoire. Cependant, l'établissement présente une originalité particulièrement intéressante. Une Galerie des dons offre l'opportunité, à qui le souhaite, de donner un objet personnel au patrimoine national français, ou même de le prêter un temps, afin d'illustrer l'histoire migratoire de sa famille. Cela contribue ainsi à construire une collection tout à fait originale. Le musée accepte tout ce qui lui est proposé, sans que le conservateur n'interfère selon ses critères esthétiques (Musée de l'histoire de l'immigration, 2014 : 9). Ce complément à l'exposition se situe dans le plus bel espace

5 On pensera notamment à l'œuvre de Kader Attia, La machine à rêve. Devant un distributeur une jeune fille est " sur le point d'acheter l'un des articles proposés [...] : un kit mariage, du gin hallal, des cartes de crédit hallal... autant de produits de notre société de consommation et du besoin de s'y reconnaître [...]. Pour le musée de la Cité, Kader Attia a imaginé une version féminine dans laquelle le distributeur propose un ensemble d'objets symboliques, représentatifs, selon l'artiste, du rêve d'intégration de certaines jeunes filles [des préservatifs, de l'alcool, des sous-vêtements strings tous estampillés "Hallal" - ndla]. Procédant par détournement, I'artiste transgresse l'objet et sa signification première et propose une réécriture du réel afin de traduire les déchirements de I'exil ; entre deux mondes, deux identités. II construit un langage particulier, à la fois poétique et politique, pour affronter la difficile équation entre désir d'appartenance à une société d'accueil et préservation de valeurs traditionnelles " (selon le cartel de l'œuvre : http://www.histoire-immigration.fr/musee/collections/la-machine-a-reve-de-kader-attia). S'il n'est pas question ici de critiquer l'œuvre, certains historiens, dont moi-même, se sont interrogés sur le pourquoi de sa présentation dans un musée de société et non un musée d'art, tant cela complexifiait clairement le message pédagogique, notamment à I'attention des jeunes publics scolaires.

6 Ancien ministre de la Culture et de la Francophonie entre 1993 et 1995.

7 Selon notre propre expérience de travail. 
du bâtiment (une mezzanine qui surplombe la magnifique salle des fêtes) et se décline en cinq sections : "Hériter ", "Partager ", "Contribuer ", "Accepter ", "Témoigner ". Et à chaque objet correspond une petite modélisation cartographique qui explique sa migration, de son départ à son arrivée en France. La section "Témoigner ", quant à elle, permet à chacun d'enregistrer son histoire, qui sera conservée par le musée, et de l'inscrire ainsi dans la mémoire nationale.

Si le musée de Hambourg réduit la complexité des faits migratoires au profit d'une narration linéaire, celui de Paris, propose un ensemble de thématiques très innovantes en lien avec la recherche scientifique, sans que pour autant les processus historiques ne soient clairement explicités. Et dans ces deux musées, qui s'opposent l'un et l'autre dans leur conception, l'histoire des mobilités reste profondément ancrée dans une approche nationale déclinée autour des thématiques du voyage, de l'arrivée et de l'intégration. Notons cependant qu'aucun des deux établissements ne tombe dans les pièges d'une approche communautariste ou essentialiste. À Hambourg, l'exposition distingue parfois les migrants juifs des autres, mais cela est inscrit dans I'histoire du centre, qui proposait une cuisine casher à ceux qui le souhaitaient. À Paris, de très nombreuses vagues migratoires venues d'Europe centrale et orientale, d'Asie et d'Afrique sont mentionnées. Mais jamais le parcours ne présente les groupes de façon autonome ; il insiste au contraire sur l'universalité de la condition de migrant et de l'expérience vécue commune.

\section{Des musées de la diaspora juive à Paris, à Berlin, à Moscou et à Ramat-Aviv}

Les musées consacrés à la diaspora juive, quant à eux, connaissent une injonction similaire à se plier à une narration historique nationale. La mobilité juive est ainsi toujours présentée dans sa dimension antique ; entraînée avec la chute du second Temple, détruit à Jérusalem en 70 de notre ère par les troupes romaines (chaque exposition permanente évoque l'arche de Titus, dans lequel les troupes romaines victorieuses défilent à Rome avec les objets cultuels du temple détruit $\left.{ }^{8}\right)$. Mais si l'événement est toujours montré comme à l'origine de la dispersion des populations juives dans le bassin méditerranéen, puis en Europe, aucun des musées visités en Europe ne fait clairement référence à la façon avec laquelle fonctionne un groupe social en situation diasporique. Pas même la définition de diaspora, traduite étymologiquement de I'hébreu comme "le tout dans un ensemble " et qui sert de point de départ aux historiens du judaïsme pour aborder la vie des Juifs en situation de minorité nationale, n'est mobilisée. Cette dernière permet pourtant de penser le groupe à la fois dans son pays de résidence et dans ses liens entretenus avec ceux installés dans d'autres pays (Tsur, 2001). Bien au contraire, le phénomène diasporique est gommé et chaque parcours montre l'apport de la culture juive à la culture de leur pays et la légitimité historique des Juifs à y vivre. II y a là une volonté d'insister, en situation post-Shoah, sur l'évident principe que les Juifs participent depuis plus d'un millénaire, à la culture et à la vie des pays dans lequel ils sont installés. Et de ce fait, les mobilités, les circulations et les migrations connues par le groupe

8 Le Musée d'art et d'histoire du Judaïsme de Paris expose la toile de Le Caravage, L'Arc deTitus, ceux de Moscou et de Ramat-Aviv reproduisent le bas-relief de la colonne de Titus de Rome. 
sont généralement minimisées, tant elles sont suspectées, au regard de la longue tradition antisémite européenne, de présenter les Juifs comme étrangers à leur pays.

Le Musée d'art et d'histoire du Judaïsme de Paris est probablement, des quatre musées visités, celui qui insiste le plus sur la dimension internationale, du moins européenne et nord-africaine de la culture juive française. Mais paradoxalement, il ne mentionne pas les faits migratoires. Installé dans l'Hôtel particulier de Saint-Aignan, construit au XVIle siècle dans le Marais, le traditionnel quartier juif de Paris, son parcours muséographique, qui date de 1998, "cite des moments précis de l'évolution du peuple juif en exil et s'appuie sur des événements majeurs qui ont marqué le judaïsme, de l'implantation des communautés en France et en Europe, à l'expulsion des Juifs d'Espagne et à ses conséquences, de l'émancipation politique des Juifs sous la Révolution française jusqu'à la Seconde Guerre mondiale " (Musée d'art et d'histoire du Judaïsme, $1999: 6$ ). L'histoire juive mise en musée rend compte du modèle français d'émancipation initié par la première Révolution française, la naissance des consistoires sous Napoléon $1^{\mathrm{er}}$, I'Affaire Dreyfus, etc. via une remarquable collection d'objets, mais les mouvements migratoires vers la France sont, quant à eux, sous-entendus. Aucune carte, ni aucun cartel ne les évoquent. Ainsi, le visiteur pénètre dans la section nord-africaine, sans que rien ne lui explique le pourquoi d'une telle présence en France ; on trouvera une brève évocation de ces mouvements migratoires de la seconde moitié du XXe siècle dans le guide des collections, mais les routes qui ont conduit les individus des anciennes colonies d'Afrique du Nord vers la métropole ne sont pas même mentionnées : " [...] d'importants mouvements migratoires se sont développés dans la seconde moitié du siècle [le $\mathrm{XXe}-\mathrm{ndla}$ ] en direction du continent américain (États-Unis, Canada, Amérique latine) et vers Israël, où les sépharades constituent désormais la majorité de la population " (Musée d'art et d'histoire du Judaïsme, 1999 : 86). L'histoire des Juifs étrangers, originaires d'Europe centrale et orientale, arrivés en France dès la fin XIXe siècle est tout aussi passée sous silence. Si le musée expose les artistes majeurs de l'École de Paris, la biographie des peintres n'insiste pas sur leur mobilité. Enfin, dans un chapitre intitulé " Être juif à Paris en 1939 ", le guide mentionne "l'immigration de Pologne, de Russie et de Hongrie " afin d'introduire le visiteur à l'œuvre de Christian Boltanski, Les habitants de l'hôtel SaintAignan en 1939, qui évoque les habitants des lieux morts en déportation (Musée d'art et d'histoire du Judaïsme, 1999 : 145). Ici encore, on ne trouvera rien sur I'histoire de l'immigration juive d'Europe centrale et orientale vers la France. Le parcours muséographique se termine par une évocation de l'État d'Israël et de I'histoire du sionisme. Mais toujours selon une même logique d'ignorance des mobilités, aucun flux, ni mouvement migratoire n'est présenté.

Le Musée juif de Berlin a, quant à lui, ouvert ses portes trois années plus tard, en 2001. Dans la capitale qui a fait le choix de muséifier son histoire récente et d'affronter dans son urbanisme la mémoire de la période nationale-socialiste, le musée à la conception architecturale extraordinaire voulue par Daniel Libeskind est une œuvre en soi (Manale, 2002). Sa muséographie aborde I'histoire judéo-allemande d'une façon identique à celle du musée parisien. Et selon le mot d'accueil de son directeur, Michael Blumenthal, ancien secrétaire d'État américain au trésor d'origine allemande, dans l'audioguide mis à la disposition du visiteur : "Le Musée juif de Berlin retrace l'histoire avec ses hauts et ses 
bas des relations entre Juifs et non-Juifs en Allemagne. L'exposition montre ce qui est possible quand des minorités religieuses, culturelles ou ethniques mettent les talents qui leur sont propres au profit de la vie nationale et quelles sont les conséquences pour tous lorsque les préjugés et l'intolérance prennent le dessus [...] ${ }^{9}$. II s'agit bien de proposer une histoire allemande du judaïsme, sans omettre ni exclusivement consacrer l'espace muséographique à l'histoire de la Shoah (Grynberg, 2003 : 155). La collection composée d'œuvres d'art, du Moyen Âge à nos jours, d'objets quotidiens et de témoignages est ici encore, particulièrement riche. Et à travers elle, les migrations juives sont directement abordées. Mais elles le sont principalement sous l'angle de l'exil subi par les Allemands qui ont pu fuir le nazisme. Le visiteur, plongé au début de l'exposition dans une architecture déroutante, et sans grande indication du chemin à suivre, croise trois couloirs, dénommés " axes " : celui de l'Exil, celui de l'Holocauste et celui de la Continuité. Et la définition donnée de l'exil dans le premier sert de fil directeur pour évoquer les mouvements migratoires connus des Juifs allemands : "En 1938, un journal juif écrivait : "Pour tous les Juifs habitant l'Allemagne aujourd'hui, la question la plus urgente est probablement : quand et où puis-je émigrer ? Entre 1933 et 1941, quelque 280000 Juifs allemands ont fui le régime nazi, rejoignant les États-Unis, la Palestine, la Grande-Bretagne et d'autres endroits du monde, tels que l'Amérique du Sud, I'Afrique et même Shanghai en Chine" ". Le guide de l'exposition, qui répertorie des objets présentés dans le parcours, mentionne lui aussi le destin de leurs propriétaires. C'est le cas, par exemple, de la pièce Drapeau à l'étoile de David de Martin Friendländer qui " en 1939 parvint à émigrer en Australie " (Musée juif de Berlin, 2010 : 26). Les mouvements migratoires expliqués dans le musée ne le sont principalement que sous l'angle de l'émigration forcée contemporaine, même si l'exposition permanente se termine par une évocation des départs en Israël et la renaissance du judaïsme en Allemagne, grâce à de nouvelles immigrations, en provenance notamment des ex-Républiques soviétiques.

Le Musée juif de Moscou, le plus récent à ouvrir ses portes, a été inauguré en 2012. Situé dans l'ancien dépôt de bus Bakhmetiev, il a été créé à l'initiative du rabbin Berl Lazar et du président de la Fédération des communautés juives de Russie. Le concept muséographique a été confié à l'agence américaine qui avait auparavant réalisé le Musée de la Shoah de Washington ${ }^{10}$. Présentant une muséographie résolument moderne, sa conception intellectuelle qui insiste sur le rôle joué par la culture juive dans la culture russe ne diffère pas, ici encore, des autres musées juifs ${ }^{11}$. Par contre, le visiteur ne voit que de rares œuvres d'art mais d'intéressants objets de culte parfois très anciens. Seules quelques sculptures monochromes d'hommes, de femmes et d'enfants participent à recréer, au centre de l'exposition et de façon fantomatique pour marquer leur absence, ce qu'était l'ambiance des shtetls. La thématique migratoire est par contre au cœur des douze sections de l'exposition permanente. Des cartes, nombreuses, localisent les lieux d'installation des communautés juives depuis le IVe siècle de notre ère. Chose plus intéressante encore, l'exposition qui ne passe sous

9 Retranscription de l'audioguide, écouté le 27 novembre 2015.

10 L'agence Ralph Appelbaum Associates (RAA).

11 Une intéressante vitrine expose, par exemple, le parallèle existant entre l'alphabet hébraïque et l'alphabet cyrillique. 
silence aucune thématique, pas même les pogroms, les assassinats antisémites commandités par Staline, la Shoah et ses dénis de reconnaissance par le régime soviétique, évoque dans sa dernière section, la migration des refusniks et les départs innombrables des Juifs de Russie en Israël et aux États-Unis à partir du milieu des années 1990. Le parcours impressionne ainsi le visiteur par sa volonté de ne rien passer sous silence et sa mise en scène, qui a su trouver un juste milieu entre émotion et narration, objective des faits. On verra ainsi la fiche signalétique de Menahem Begin, photographié de face et de profil, lors de sa déportation au goulag en 1942 (Jewish Museum and Tolerance Centre, 2014 : 161). Un beau film, relatant I'histoire de la Seconde Guerre mondiale fait ainsi intervenir de très nombreux témoins à la parole affranchie de toute contrainte politique, en complément à une narration en voix-off décrivant les événements chronologiques.

Ces trois établissements se situent intellectuellement en opposition avec le Musée de la diaspora (littéralement la Maison de la diaspora - Beit Hatefutsoth) de Ramat-Aviv en Israël. Là où les musées européens montrent que les Juifs sont membres des nations dans lesquelles ils résident, celui d'Israël, dans sa dimension nationaliste, insiste au contraire sur la légitimité des Juifs à venir vivre dans le pays et signe ici " la fin de l'histoire " de 2000 ans de vie " en exil ". Ce dont témoigne le panneau introductif à l'exposition, une citation du poète Abba Kovner : " C'est I'histoire d'un peuple qui a été dispersé dans le monde entier et qui pourtant est resté une même famille. Une nation qui a été maintes et maintes fois condamnée à la destruction et une fois encore, sur les ruines, a construit une nouvelle vie ". Le guide de l'exposition est encore plus explicite et ouvre ses pages par une citation attribuée au rabbi Nahman de Bratslav : "Là où je vais, je me rends en terre d'Israël " (Beit Hatfutsot, sans date : 10). Et sans surprise, les faits migratoires sont particulièrement bien représentés dans le musée en prenant soin de ne pas les réduire à leur seule dimension ashkénaze (Trevisan-Semi et al., 2013 : 2). Son parcours muséographique est ici historique, tout en insistant fortement sur la thématique religieuse. Là où la religion était expliquée dans les musées européens, elle est ici évoquée dans sa dimension quotidienne de garante de la conservation du judaïsme (les fêtes principales et secondaires, les mariages et les actes rituels sont illustrés). Et contrairement aux autres musées juifs visités, le parcours permanent insiste sur la forte dimension migratoire de I'histoire juive. II s'ouvre bien évidemment avec I'histoire des conquêtes assyriennes, babyloniennes et romaines qui ont entrainé l'exil juif et se poursuit par la description de la vie en diaspora. Le musée mentionne les documents de Genizah, qui de 870 à 1880 donnent à voir ce que fut la vie juive au Caire ; le livre de voyage de Benjamin de Tudela, qui au XIle siècle a commenté son périple le menant d'Espagne en Perse ; la vie en Europe après les expulsions de la Péninsule par les rois chrétiens; les routes du commerce international organisé depuis et vers Amsterdam et l'installation de communautés juives en Europe centrale et orientale. Et toujours selon la même logique diasporique, les trajectoires du XIXe siècle menant aux continents américain et australien sont cartographiées. Enfin l'exposition se termine par l'évocation de la loi du retour en Israël et douze portraits biographiques (dont trois femmes) d'Israéliens, résumant à la fois les multiples origines géographiques et ethniques des Juifs 
et les différentes vagues d'aliya qui se sont succédé ${ }^{12}$.

Les parcours permanents de ces expositions proposent une définition des migrations très naturalisée, réduite selon le sens commun à des trajectoires collectives, au cours desquelles les individus quittent un lieu pour un autre, en traversant une ou plusieurs frontières nationales. Observer les phénomènes migratoires à l'échelle macroscopique permet en effet au concepteur d'exposition de trouver plus facilement les illustrations nécessaires à son propos : cartes générales de géographie, documents d'archives et de presse, focus sur des événements précis, etc. quitte à ne pas prêter grande attention à une observation mésoscopique qui rendrait compte de trajectoires genrées, de groupes plus modestes en nombre, des circulations et des formes prises dans les installations. Cela ne signifie cependant pas pour autant que les approches microscopiques sont ignorées. Bien au contraire, le témoignage et l'expérience individuelle sont toujours au cœur des propos exposés. Mais c'est bien le jeu d'échelle, entre les approches macro, micro et méso qui est absent de ces musées, et qui ne permet pas d'avoir une vision complexifiée de ce que furent les faits migratoires.

\section{Un « objet migration " naturalisé}

Sans surprise aucune, on ne trouve dans ces expositions ni citation des ouvrages historiques devenus les "classiques des études de sciences sociales", ni références bibliographiques et historiographiques présentant comment l'étude dans le temps et dans l'espace des mobilités est devenue nécessaire à la compréhension du monde contemporain. Bien au contraire, les migrations sont réduites à des événements historiques "isolables ", dont la cartographie vient toujours en résonnance avec la très classique théorie du push and pull, qui à elle seule est supposée expliquer toutes les formes de mobilités.

\section{De points et de flux. Où une cartographie classique est réduite à sa fonction illustrative}

Les expositions permanentes visitées présentent toutes des cartes générales de géographie, la plupart réalisées pour ces expositions ${ }^{13}$. Si elles illustrent les flux et les routes empruntées par les migrants, de leur point de départ jusqu'à celui de leur arrivée, elles ne sont généralement pas mobilisées pour comprendre des pratiques migratoires. Les multiples étapes connues par les individus au cours du voyage, les détours, les installations temporaires en un lieu avant de poursuivre la route, les circulations ne sont pas pris en considération. Et de fait, la cartographie présentée est loin d'être innovante. Elle laisse de côté toute idée de construction dans le temps des champs migratoires, ignore la répartition des individus dans les pays, leurs circulations et aller-retour. De même, elles ne sont pas discrétisées selon le genre, les âges, etc. Elles sont,

12 Aliya (plur. Aliyot), littéralement " la montée en Israël " en hébreu, est le terme qui décrit l'immigration juive vers Israël. On lit ici des notices biographiques d'un homme dont la famille a toujours vécu " sur la terre d'Israël " et d'originaires du Kurdistan, de Cochin, de l'Oural, du Maroc, du Yémen, de Lituanie, de Rome (deux cas), d'Allemagne, des USA et d'Espagne.

13 Le Musée d'art et d'histoire du Judaïsme de Paris, ne présente qu'une seule carte, reproduction de I'Encyclopaedia Judaica, traitant des trajectoires des Juifs ibériques après les expulsions de la fin XVe siècle. 
pour la plupart d'entre elles, des projections de Mercator, européocentrées, avec une sémiologie réduite a minima sur lesquelles les échelles, les effets de seuils et les légendes ne sont pas toujours marqués. Sans innovation, sans même utiliser des cartographies plus audacieuses comme les anamorphoses, l'usage de la carte fait par ces musées apparaît plus comme une illustration éventuelle qu'un objet susceptible d'apporter un savoir.

Le Musée de l'immigration parisien présente en avant-propos à son exposition permanente, un ensemble cartographique de grande qualité. Sur un très petit écran de télévision défilent une dizaine de cartes, de très belle facture, qui exposent des flux migratoires internationaux, de la fin du XIXe siècle à nos jours et accompagnés de courtes phrases explicatives (Le temps des voisins, 1891, Migrations vers les Amériques, fin XIXe début XXe, etc. $)^{14}$. On regrette cependant qu'elles restent situées en marge de l'exposition et que le lien entre migrations internationales et migrations en France ne soit pas clairement établi. Leurs titres parfois non expliqués (Système migratoire contemporain fin XXe siècle, Réfugiés et déplacés internes fin 2010 [sur une carte à l'échelle de la planète]) montrent qu'elles sont en résonnance avec la production scientifique, mais leur défilement rapide ne permet pas de saisir la subtilité du propos et le visiteur ne $s^{\prime} y$ arrête malheureusement pas ${ }^{15}$. Inversement, à Hambourg, la cartographie est simplifiée, ludique et se situe au cœur de l'exposition. Une importante installation vidéo, bilingue, consiste en la projection de flux sur un écran en forme de livre ouvert de deux mètres carrés environ (Ballinstadt Museum, sans date : 18). La carte interactive présente les pays de naissance des émigrants qui ont transité par l'île de Veddel. C'est aussi une carte interactive, en anglais, qui expose dans le Musée juif de Berlin les lieux d'exil connus par les Allemands. La très belle installation propose au visiteur de sélectionner un pays qui a reçu des réfugiés sur un planisphère (ils sont signalés par leur drapeau sur un fond de carte géopolitique) ; une fois la flèche positionnée, défilent les informations nécessaires à la compréhension de l'histoire des réfugiés allemands : une présentation de la politique générale que le pays d'accueil a adoptée avant et pendant la Seconde Guerre mondiale est accompagnée de quelques illustrations. La carte n'hésite pas non plus à dire l'absence de données historiques. Le curseur sur la Palestine mandataire entraine ici le défilement d'un texte en style télégraphique : "Important refugee destination ++ Immigration quotas determined by British Mandate Government " et apparaît en marge la photographie d'une femme allemande prise dans les paysages de Galilée. Pour la Principauté de Monaco, le texte est plus lacunaire : "Unimportant as a country of exile ++ Number of Jewish Refugees unknown ". Dans le cas de la France, les données sont évidemment nombreuses : "Most important exile country in Europe ++ Visa required ++ Increasingly restricting immigration policies ++ Extraditions ++ Internment at the start of the War ++ Expulsions and Deportations // Several Escapes across the Borders ". À ce texte simple, mais efficace, viennent se joindre une reproduction d'une peinture d'Arbit Blatas, Le café du Dôme, présenté comme étant un lieu parisien de sociabilité des réfugiés,

14 Cf. http://www.histoire-immigration.fr/histoire-de-l-immigration/questions-contemporaines/cartes. Le site internet du musée reprend les cartes diffusées sur l'écran, en quinze sections.

15 Selon nos observations réitérées à plusieurs reprises. Dans les autres musées visités, les cartes retiennent I'attention des visiteurs. 
des artistes et des intellectuels, ainsi que la photographie prise en 1939 d'une femme allemande assise avec ses enfants devant un wagon de train dans une gare de la capitale. C'est aussi une très importante installation cartographique qui accueille le visiteur du Musée juif de Moscou. Des cartes défilent sur un globe, selon le séquençage historique du parcours muséographique, montrant les étapes d'installation des Juifs dans la région, du IVe siècle à la fin de l'époque moderne. Par ailleurs, chaque étape du parcours muséographique s'ouvre avec une ou plusieurs cartes, punctiformes ou de flux, précédemment présentées et qui exposent les caractéristiques géohistoriques (localisations, création de villes, etc.) de la période. Ces cartes, de grande qualité, sont reproduites dans le livret de l'exposition. Au Musée de la diaspora encore, de nombreuses cartes viennent illustrer chaque étape du parcours permanent. Elles peuvent être grandes en taille, ou plus petites. Et bien que de facture ancienne - elles auront bientôt quarante ans - elles sont au cœur de la démonstration désirée. Elles exposent les trajectoires et les routes migratoires empruntées à chaque période.

Ainsi, et sans surprise, la carte exposée " pour elle-même " ne retient pas I'attention du visiteur. Inversement, lorsque la muséographie la rend attractive, en l'incluant dans le cœur de l'exposition, où en l'entourant d'une installation ludique, elle vient en complément de la médiation faite par l'art, l'objet ou le texte. Cependant, la cartographie est généralement peu originale, pensée comme devant illustrer des flux, et non pas des dynamiques historiques. Et si la modélisation cartographique reste de facture classique, il n'est pas vraiment étonnant que le phénomène migratoire soit aussi abordé de façon très traditionnelle. Et ce dernier est principalement expliqué par les grandes causes susceptibles de répondre à la question " du pourquoi des départs".

\section{Of Push and Pull. Où les causes des migrations sont préférées à l'étude des projets migratoíres}

Dans la première salle du musée hambourgeois, de grandes sphères de plastique installées au plafond présentent les rêves des émigrants. "Argent ", "liberté " sont les principaux mots-clefs inscrits en bilingue sur l'installation. Et la question des départs est ici classiquement posée, soutenant le mythe d'une quête de l'eldorado : "Pourquoi les gens partent-ils ? [...] La raison plus commune pour émigrer était le souhait d'une plus grande stabilité économique, quand elle n'existait pas dans les pays d'origine. De mauvaises récoltes entraînant des famines ont renforcé le désir de chercher une nouvelle patrie, avec des perspectives d'avenir plus prometteur. [...] Les persécutions politiques et religieuses ont aussi poussé les individus à quitter leur pays d'origine, en espérant que leurs croyances seraient tolérées à l'étranger " (Ballinstadt Museum, sans date : 16). Dans le musée parisien, les causes données à l'émigration sont identiques, mais avec une exhaustivité des exemples historiques. La recherche d'emploi est illustrée par les saisonniers belges de 1840 et les Italiens d'avant la Première Guerre mondiale. Les difficultés politiques par les révolutionnaires polonais de 1831, les Juifs victimes des pogroms après 1881 et, entre autres, les réfugiés de la guerre civile espagnole. L'exposition présente ainsi les magnifiques photographies de Robert Capa et de David Seymour. Et pour chaque vague migratoire dans un souci d'exhaustivité, celle des Algériens, des Portugais, des réfugiés du Sud-Est asiatique, d'abondantes illustrations sont visibles. Dessins et photographies de presse, documents d'archives et objets sont ici en profusion. 
Les facteurs de "pull " sont présentés dans la même logique. Dans le musée de Hambourg, le passage de la frontière de l'État d'accueil est montré comme une épreuve. Le visiteur peut, s'il le souhaite, répondre au même questionnaire auquel étaient soumis les migrants à Ellis Island. Mais une fois rentré sur le territoire, il expose combien les opportunités professionnelles sont présentes (la crise économique de 1929, est passée sous silence). Le Musée de l'histoire de l'immigration de Paris, quant à lui, montre I'histoire du droit de travail et du passage des frontières : il expose ainsi les différentes périodes qui se sont succédé : les entrées libres et les séjours contrôlés d'avant 1914, puis I'histoire d'un État devenu tour à tour recruteur, répressif (avant et sous le régime de Vichy), puis tentant une maîtrise des flux à partir de 1974. On trouvera dans cette section I'histoire des réfugiés, des Arméniens aux " harkis " notamment et les politiques de régularisations qui se sont succédé depuis le milieu de la décennie 1970. Ici encore, de nombreux documents sont exposés : passeports Nansen, dessins de presse, photographie du hall de Sangatte, etc. Mais une fois la frontière passée, le visiteur est plongé dans l'histoire française de deux siècles de travail, de l'essor industriel à la crise d'aujourd'hui. Le musée évoquera la question des luttes sociales. Les documents insistent sur les opportunités offertes par la France : travail industriel, niches professionnelles dans le textile, la sidérurgie et l'exploitation minière. Le monde du salariat et de la boutique sont ainsi évoqués, au travers des travailleurs et de grandes sagas familiales (Cité nationale de l'histoire de l'immigration, 2009 : 140-167). Une petite section a aussi été récemment ajoutée portant sur les migrations des élites.

Les musées juifs de Berlin, de Moscou de Ramat-Aviv, insistent, on l'a vu, beaucoup plus sur le " push " politique et les persécutions perpétrées par les régimes nazi et soviétique. Un rare film amateur montre l'incendie de la synagogue de Bielefeld en 1938. De nombreuses photographies évoquent les marquages opérés par les nazis des magasins. À Moscou, le musée présente I'histoire du sionisme soviétique, de la culture juive " underground " sous la dictature, aux revendications à I'aliya des refuzniks en lien avec la Perestroïka. Les photographies illustrent notamment les manifestations importantes de 1987, les départs des années 1990 et l'accueil de ces migrants en Israël par Yitzhak Rabin (sans date) ou le premier ministre Ehud Barak en 2000. Le Musée de la diaspora de Ramat-Aviv enfin, insiste peu sur les causes de l'immigration en Israël, quand elles ne sont pas politiques. Et les multiples projets à l'origine des Aliyot sont ignorés, au profit du rappel du droit, sinon " du devoir " de chaque juif à venir vivre en terre d'Israël.

\section{L'intégration et le retour. Où la circulation du migrant est minorée}

L'intégration des migrants n'est pas un phénomène décrit dans ses processus de construction, mais reste présentée comme une évidence qui vient clore la route migratoire. Le musée de Hambourg expose ainsi comment, les immigrés ont recréé, à New York, leur environnement familier. La vitrine d'une " typique librairie allemande " expose un "dictionnaire pour les migrants " et insuffle ici l'idée d'un apprentissage immédiat de la langue du pays d'accueil. II rappelle que les " beer-gardens ", nombreux, favorisaient les sociabilités. Le livret précise cependant qu'après le naufrage d'un bateau de plaisance en 1904 et le décès de 1100 victimes résidentes de "Little Germany ", le quartier a été déserté et investi 
par des Juifs originaires d'Europe de l'Est (Ballinstadt Museum, sans date : 51), ce qui explique aujourd'hui que la topographie new yorkaise n'a pas retenu, contrairement à China Town ou Little Italy, I'origine nationale d'individus qui le peuplaient. Au terme d'une période d'adaptation, l'exposition précise que les Allemands sont devenus, en une génération, des Américains. Une intéressante carte postale, reproduite dans le livret, montre un jeune enfant brûlant dans une cheminée des jouets de bois fabriqués en Allemagne, illustrant ainsi, durant la Première Guerre mondiale, " comment il coupe le cordon avec leur ancienne maison " (Ballinstadt Museum, sans date : 51 ). Et les liens entre pays d'origine et descendants d'immigrants sont littéralement absents : les tentations nationales socialistes de l'Entre-deux-guerres ne sont absolument pas évoquées.

Le Musée de l'histoire de l'immigration de Paris insiste, quant à lui, sur les formes d'intégration, sans jamais prononcer le mot, lui préférant celui " d'enracinement ". La scolarisation des enfants, les luttes syndicales, le sport, la participation aux guerres mondiales et à la Résistance sont autant d'éléments illustrant I'intégration des immigrés, au côté des Français. Cet inventaire des luttes communes insiste ainsi sur " deux siècles d'une histoire partagée " (Cité nationale de I'histoire de l'immigration, 2009 : 195). Et dans cette logique de rapprochement des groupes, sinon de fraternisation, le visiteur est aussi informé de la longue histoire des luttes politiques, et notamment contre le racisme et I'antisémitisme, menées conjointement par Français et immigrés. Le parcours présente enfin, dans un prolongement logique, des certificats de naturalisation, sans ignorer les freins politiques et administratifs qui ont pu exister pour leur obtention.

Dans ces deux expositions, la circulation entre pays d'adoption et pays de naissance reste sous-entendue. Le musée de Hambourg évoque ainsi que si un migrant ne trouve pas sa place dans le pays d'accueil, il pourra toujours " rentrer " dans son pays. Le livret assure ainsi qu'en dépit de statistiques peu fiables, et sans aucune précision de période historique, ce fut le cas de $20 \%$ des immigrants (Ballinstadt Museum, sans date : 52). La muséographie présente ainsi quelques photographies de mendiants et de soupes populaires à la fin du XIXe siècle, mais illustre de préférence le retour par la narration de deux success-stories : celle d'un chercheur d'or ayant fait fortune en Idaho et d'une femme qui se maria avec un des membres de l'équipage du bateau rencontré lors de son départ vers les États-Unis. Le Musée de l'immigration, de son côté, évoque de façon très impressionniste la circulation par un cartel explicatif rappelant " que les retours sont fréquents pour ceux qui le peuvent " et qu'il est toujours possible de rentrer après " la fin du régime d'oppression pour les exilés, volonté de participer à la renaissance du pays d'origine, promesse de travail ou nostalgie "(Cité nationale de I'histoire de l'immigration, 2009 : 94). Enfin, c'est une œuvre de Thomas Mailaender, Les voitures cathédrales, mettant en scène les automobiles surchargées prêtes à prendre la route qui sera la plus visible pour exposer cette question (Cité nationale de l'histoire de l'immigration, 2009 : 110-111). Le "va-et-vient identitaire ${ }^{16}{ }^{16}$ complémentaire à ces mobilités et jamais mentionné comme tel, est brièvement évoqué par la recréation des sociabilités nationales, les pratiques religieuses et linguistiques conservées, mais

16 Expression reprise de Charbit, Hily et Poinard (1997). 
aussi adaptées à l'aune des évolutions culturelles connues au contact du pays $\mathrm{d}^{\prime}$ accueil. Cette approche est identique à celle des musées d'histoire juive, où la culture d'origine, par un effet d'osmose, est présentée comme venant enrichir la culture nationale des pays hôtes. Ce qui participe à donner du migrant l'image d'une personne en constante voie d'intégration.

La migration est ainsi exposée comme étant un acte collectif, isolable par ses causes, ses flux, et ses trajectoires. Partir n'est pas pensé comme relevant d'un déroulement simple, ni une opportunité. Bien au contraire, c'est une histoire de groupes et de moments qui est ici mise en avant. II n'y a aucun espace dédié à une présentation des concepts de projet migratoire, d'autonomie, de mobilité. La notion de circulation est, quand elle présentée, particulièrement minimisée. Et c'est dans le seul récit des expériences migratoires, que l'on trouvera la dimension individuelle jusqu'alors manquante et une nécessaire nuance pour la compréhension de ce qu'est l'acte de migrer.

\section{L'originale muséographie des expériences migratoires}

Les témoignages sur les expériences vécues sont bien évidemment au cœur de la muséographie et permettent une sensibilisation " au monde invisible des migrants ". On les trouve dans de nombreuses archives d'État, dans les narrations faites par les individus ou leurs descendants, et dans le cas du temps présent, par des témoignages audios et visuels. Et le recours à ces supports et à ces exemples individuels mais paradigmatiques favorise des effets de proximité.

\section{Le témoignage. Une explication convenue, mais rare}

Le musée de Hambourg propose ainsi, sur des bornes audios, trois témoignages de quelques minutes, disponibles en allemand et en anglais. Une actrice prête sa voix à la narration de l'expérience connue par " une paysanne autrichienne émigrant avec sa famille dans les années 1860 ". D'autres témoins se racontent, longtemps après leur voyage : " un écolier émigrant avec sa famille vers 1905 " et " une femme juive allemande fuyant les nazis avec son époux et ses enfants en 1938 ». Ces textes sont courts et donnent aux visiteurs l'essentiel de ce que fut un parcours : les causes du départ, les souvenirs marquants du voyage et l'arrivée. Mais les détails sont absents. Et la mobilisation de l'histoire orale ne présente pas particulièrement l'expérience migratoire. Le témoignage ici, est en parfait accord avec la définition très réduite et normative de l'immigration qui est donnée dans le musée.

On ne trouve pas dans le musée parisien, de témoignages sur l'expérience migratoire en elle-même ; et les mémoires recueillies par les équipes du musée ne sont pas mobilisées dans l'exposition. Dans les musées juifs enfin ce sont les événements tragiques de la Shoah qui sont donnés à l'écoute. Aussi, le recueil de la parole qui est au cœur de l'activité des chercheurs et des services de conservation des archives et bibliothèques nationales de chacun des pays n'est pas un réflexe muséal évident. Leurs enregistrements, leurs rapports, ne sont pas présentés, alors qu'ils mettent en lumière les aspérités de l'acte de migrer, ajoutent de la complexité dans une approche trop souvent lissée. Les musées ne prêtent que très peu d'attention à l'énonciation directe de l'expérience, aux 
mobilités et, de façon générale, ne viennent pas en complétion des archives exposées. Ce sont au contraire des objets ou des œuvres qui sont les motifs de narration et qui entraînent un dialogue concret entre le visiteur et le migrant.

\section{Le récit par l'intercession de l'objet. L'émotion et la catharsis mises en scène}

C'est le Musée de I'histoire de l'immigration à Paris qui laisse la plus grande place à l'objet. II y est pensé comme un révélateur des expériences migratoires et occupe la double fonction de patrimoine de l'immigration et de catalyseur d'émotions. La pièce présentée n'est pas forcément esthétique. Elle peut être imposante ou petite, très abimée ou inutilisée, mais elle permet de révéler les sentiments que les individus qui en étaient les propriétaires ont projetés sur elle. Agencés dans de vastes vitrines ces objets sont accompagnés d'un cartel informant des motivations du don et de l'histoire migratoire de son propriétaire. Une petite modélisation présente de façon complémentaire les trajectoires qui furent celles de la personne. Et l'objet permet ainsi d'évoquer la dimension affective et psychologique de l'immigration.

La Galerie des dons expose ainsi la truelle de plâtrier du père de François Cavanna. Son témoignage évoque l'attachement de son propriétaire et de façon plus objective, en quoi il symbolise l'immigration des travailleurs italiens originaires de la Vallée de l'Apennin (Musée de I'histoire de l'immigration, 2014 : 190-191). Plus imposante en poids, une fraiseuse est supposée témoigner de I'histoire d'Emilio Reig, réfugié espagnol (Mazaubrun, 2012) : "Une tonne d'acier, c'est à la mesure de tout ce qu'on a apporté à la France en tant que fils et filles de républicains espagnols (sic). Grâce à cette machine, mon père pouvait se sentir utile. Elle représente sa fierté d'ouvrier et son savoir-faire " (Musée de I'histoire de l'immigration, 2014 : 153). Et selon un même principe, le musée parisien expose un babyfoot qui a servi lors d'une occupation des lieux par des militants sans-papiers, un costume de danse orientale, la valise d'un militant des droits I'Homme, etc. Tous ces objets ont pour fonction d'injecter une dimension individuelle à la définition de l'immigration mais aussi, de façon impressionniste, de rappeler la dimension militante du musée.

Ce sont aussi des objets cathartiques qui illustrent les départs des Juifs allemands sous le régime nazi. Dans l'avant-dernière section de l'exposition "National Socialism 1933-1945 ", la muséographie interpelle le visiteur en le confrontant aux expériences vécues par les réfugiés : "What would you take along if you had to leave your country? ", propose ainsi un des panneaux introductifs. Le visiteur peut alors soulever quatre caches qui dévoilent un objet emporté mais aussi la trajectoire et la photographie de son propriétaire. Herbert Simon a quitté I'Allemagne pour Valparaiso au Chili en 1939. II a fait don au musée de la boussole qu'il avait apportée. Le symbole est fort et illustre la difficulté qui fut sienne à trouver de nouveaux repères. Alice Schindel partie en 1940 aux États-Unis rejoindre son père, via un long périple qui I'a conduit avec sa mère à Moscou et en Chine, a emporté avec elle un recueil de poésies : "Grâce à ce livre, Alice était toujours en mesure de se souvenir de ses amis et de ses camarades de classe ". C'est la rupture avec un monde ordinaire qui est ici mise en scène. Rudi Librowicz a, quant à lui, quitté enfant I'Allemagne, en 1937. II 
prend avec lui un petit modèle de suka17. La notice biographique précise : " II a changé son nom de famille en Leavor, comme les Anglais avaient des difficultés à prononcer Librowicz ". La perte de la culture, et la religion comme principal moyen de conserver son identité, sont ici mises en scène. II était très difficile pour des parents d'envoyer leurs enfants seuls dans un pays étranger. Max Heymann écrivit ce poème pour réconforter sa fille Éva. II I'intitula À l'occasion du 20 mai 1939, jour du départ d'Éva pour l'Angleterre. II termina son poème par ces lignes : "Le vieux monde nous a déçus. Que ta vie renaisse ". Éva devint chanteuse d'opéra. La cache soulevée présente la page dactylographiée du poème. Devant cette promesse pour des jours meilleurs, le visiteur s'associe à la douleur d'un père, probablement disparu dans l'enfer de la Shoah, à se séparer de sa fille envoyée dans un monde incertain. Et à la suite de l'expérience de déchirement connue par des parents, c'est la violence d'un départ d'un enfant seul qui est enfin mise en scène. "Avez-vous un animal en peluche à la maison ? Ce singe appartenait à Gert Berliner et était toujours accroché au porte-bagage de son vélo. En 1939, Gert a été obligé de partir en Suède sans sa famille. II n'avait pas la possibilité de prendre beaucoup de jouets avec lui, mais il rangea le petit singe dans sa valise. Gert l'a conservé avec lui-même adulte, tant il lui rappelait son enfance en Allemagne ". Aussi, chacun des visiteurs, qu'il soit enfant, jeune homme ou parent est ainsi en situation de s'associer à une histoire individuelle et à une trajectoire migratoire douloureuse faite de déchirements, d'exil et de séparations avec des individus dont on sait rétrospectivement qu'ils sont condamnés.

\section{Le récit par l'œuvre d'art contemporain. Une narration implicite des migrations illégales}

Enfin, le dernier ressort des muséographes pour évoquer l'expérience en migration est le recours à l'art contemporain. Ce choix fut principalement celui du Musée de l'immigration de Paris dont l'objectif est de susciter des émotions pour appuyer son propos, quitte à parfois gommer le message scientifique. Dans un musée qui offre une profusion d'œuvres, deux délivrent le message de l'expérience migratoire. L'une évoque les voyages effectués, l'autre la question de la régularisation administrative. Bouchra Khalili propose au travers de son œuvre Mapping Journey, une illustration des trajets migratoires contemporains, les zones frontalières et les existences clandestines ${ }^{18}$. II s'agit d'une installation vidéo dans laquelle des migrants font le récit de leur migration clandestine et en complément, huit sérigraphies proposent une cartographie de leur trajectoire, présentée comme une constellation d'étoiles, dans l'espace méditerranéen. Selon la fiche signalétique de l'œuvre : "Le dispositif vidéo [...] repose sur un plan fixe d'une grande frontalité où se révèle une "contre-géographie" fondée sur la parole et le geste. En faisant le récit de leur voyage et en le dessinant, des migrants confrontent leur parcours singulier à la normativité des cartes, révélant ainsi une autre cartographie, souterraine et invisible, qu'esquissent les trajectoires migratoires contemporaines ". Les expériences par les individus interviewés sont clairement mises à nue. L'artiste Ghazel, quant à elle, met en

17 La cabane traditionnelle construite lors de la fête de Souccot.

18 Présentation de I'œuvre Maping Journey (cf. http://www.histoire-immigration.fr/ musee/collections/the-mapping-journey-project). 
scène son expérience personnelle de " sans-papier " dans l'œuvre Urgent ${ }^{19}$ (cf. Illustration 1). Après avoir reçu une lettre d'expulsion, elle " décide de mettre des annonces pour un mariage blanc [...] ". L'artiste poursuit son projet, déclinant les affiches en plusieurs versions jusqu'en 2002, date à laquelle elle reçoit une carte de résidence pour dix ans. Elle renverse alors les données et propose à son tour un mariage à un clandestin afin qu'il puisse obtenir une carte de séjour. Si son travail emprunte au registre de l'humour, de la dérision et du détournement, il dépasse la propre expérience de l'artiste et confère à l'œuvre une portée universelle : "Partout dans le monde il y a des gens délocalisés qui essayent de s'enraciner ailleurs " (Cité nationale de l'histoire de l'immigration, 2009 : 68).

Si ces travaux ne sont pas aisés de prime abord, ils permettent cependant de montrer par le recours à une biographique artistiquement traitée le projet migratoire et son évolution, tel qu'il peut être conceptualisé par les chercheurs, et les difficultés administratives de l'installation.

Illustration 1 : Lithographie (extrait) de l'œuvre de Ghazel, Urgent.

Quand l'artiste libère la parole convenue des comités scientifiques
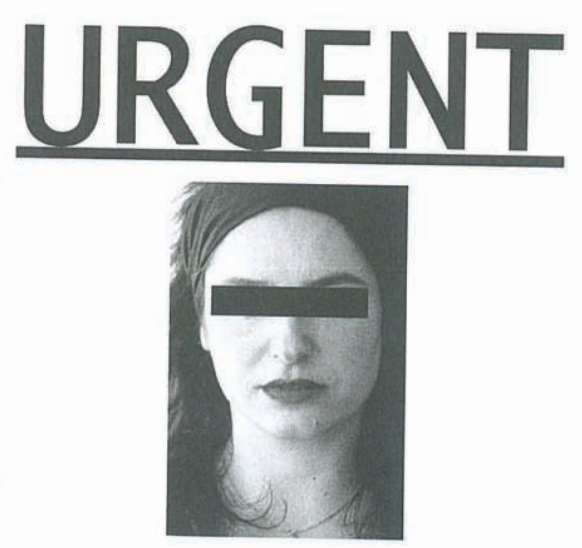

Femme, 40ans

$$
\begin{gathered}
\text { (artiste, moyenne-orientale, } \\
\text { ex-S.P.F.* \& } \\
\text { R.D.D.** en France) } \\
\text { offre marriage (papiers) } \\
\text { à un Homme S.P.*** } \\
\text { (tout origine/religion possible) } \\
\text { emait : maraateminitel net } \\
\ldots \text { : sans papiers fixe } \\
\cdots \text { résidente duré déterminée } \\
\cdots: \text { sans papiers }
\end{gathered}
$$

Source : Guide de l'exposition permanente (2009 : 69).

19 Présentation de I'œuvre Urgent (cf. http://www.histoire-immigration.fr/musee/collections/urgent-de-ghazel). 
Le message porté par ces œuvres n'est cependant que de la responsabilité de l'artiste. "Cette couverture " permet l'évocation de situations politiques complexes à aborder frontalement dans un musée national. Ce ne sont ni militants, ni discours officiels, qui évoquent les situations illégales toujours délicates à officialiser dans un musée dont les fonds dépendent directement de l'État, mais une parole artistique libre. En achetant une œuvre et en l'exposant, l'institution contourne la parole d'État et laisse à la seule parole artistique la responsabilité de propos tabous. Par cette contorsion, le musée acquiert une liberté d'expression et évite un lissage des discours. Cela entraîne cependant des conséquences muséographiques complexes. L'insertion de l'œuvre dans le parcours permanent peut parfois difficilement entrer en résonnance avec la section dans laquelle elle est exposée.

\section{Conclusion}

Ces musées de société consacrés à l'histoire des migrations et à la diaspora juive ont contribué à asseoir des discours inédits dans les paysages muséaux à l'attention " d'un grand public ". En déconstruisant les " imaginaires " xénophobes, ils montrent combien les migrations sont partie intégrante des histoires nationales. Mais les expositions qu'ils proposent ont cependant tendance à " naturaliser " l'acte de migrer et à le réduire à un déplacement linéaire, et de façon identique, à borner la diaspora à sa seule dimension nationale. Les muséographes, qui ont souvent le dernier mot dans la conception de l'exposition, préfèrent ainsi recourir à l'émotion pour retenir l'attention du public qu'à une explication objective des faits ce qui, in fine, gomme la complexité apportée par les chercheurs et ne permet pas toujours une meilleure compréhension des phénomènes migratoires actuels qui sont pourtant, depuis plus d'une décennie, au cœur des préoccupations politiques. Entre ignorer l'expérience migratoire, comme c'est le cas du Musée d'art et d'histoire du Judaïsme parisien, ou vouloir en faire une évidence avec l'exemple du musée de Ramat-Aviv, entre désirer faire ressentir la violence de l'exil à Hambourg et à Berlin, ou à vouloir trop dire, comme l'illustre le Musée de l'immigration parisien, le visiteur devra réellement poursuivre sa visite par des recherches individuelles. Et en ce sens-là, ces musées assurent une véritable fonction pédagogique.

\section{Références bibliographiques}

Ballinstadt Museum (sans date) Ballinstadt, The Emigration Museum, Hambourg, Ballinstadt Museum, $63 \mathrm{p}$.

Beit Hatfutsot (sans date) Museum of the Jewish People: Beit Hatfutsot, Tel-Aviv, Beit Hatfutsot, $54 \mathrm{p}$.

Charbit Yves, Hily Marie-Antoinette et Poinard Michel (1997) Le va-et-vient identitaire, migrants portugais et villages d'origine, Paris, INED-PUF, $144 \mathrm{p}$.

Cité nationale de l'histoire de l'immigration (2009) Guide de l'exposition permanente, Paris, Gallimard, 255 p.

Green Nancy (2011) Construire une collection, représenter l'immigration : la Cité nationale de l'histoire de l'immigration, Hermès, La Revue, 61 (3), 61, pp. 131-137. 
Green Nancy (2002) Repenser les migrations, Paris, PUF, 139 p.

Grosfoguel Ramon, Le Bot Yvon et Poli Alexandra (2011) Intégrer le musée dans les approches sur l'immigration, vers de nouvelles perspectives de recherche, hommes \& migrations, 1293, pp. 6-11.

Gruson Luc (2011) Un musée peut-il changer les représentations sur l'immigration?, hommes \& migrations, 1293, pp. 12-21.

Grynberg Anne (2003) Du mémorial au musée, comment tenter de représenter la Shoah ?, Les Cahiers de la Shoah, 7, pp. 111-167.

Jewish Museum and Tolerance Centre (2014) Atlas of Russian Jewish History based on Jewish Museum and Tolerance Centre Materials, Moscou, Knizhniki, $304 \mathrm{p}$.

Manale Margaret (2002) Berlin capitale : la ville comme exposition, L'Homme et la Société, 3, pp. 67-88.

Mazaubrun Hélène (du) (2012) Une tonne d'acier pour se forger une identité, hommes \& migrations, 1299, pp. 98-104.

Meza-Torres Andrea (2011) La muséification de la migration à Berlin et les débats sur la représentation, hommes \& migrations, 1293, pp. 28-39

Musée d'art et d'histoire du Judaïsme (1999) Guide des collections, Paris, Ministère de la Culture et de la Communication, 160 p.

Musée de I'histoire de l'immigration (2014) Guide de la Galerie des dons, Paris, Cité nationale de l'histoire de l'immigration, 254 p.

Musée juif de Berlin (2010) Le Musée juif de Berlin. Les essentiels, Berlin, Musée juif de Berlin, $174 \mathrm{p}$.

Poli Marie-Sylvie et Idjéraoui-Ravez Linda (2011) Des musées et des expositions dans le débat sur l'immigration en France, Hermès, La Revue, 61 (3), pp. 138-143.

Renart Isabelle et Poinsot Marie (2011) La place de I'art contemporain à la cité. Entretien avec Jacques Toubon, président du conseil d'orientation de la Cité nationale de I'histoire de l'immigration, hommes \& migrations, 1293, pp. 22-26.

Trevisan-Semi Emmanuela, Dario Miccoli and Tudor Parfitt (2013) Memory and Ethnicity, Ethnic Museums in Israel and the Diaspora, New-Casttle, Cambridge Scholar, $250 \mathrm{p}$.

Tsur Yaron (2001) A Torn Community: The Jews of Morocco and Nationalism 1943-1954, [in Hebrew: Yehudei Maroko ve-ha-Leumiyut 1943-1954], Tel-Aviv, Am Oved, 520 p. 


\section{Yann Scioldo-Zürcher}

\section{Les migrations au musée ! Sciences sociales et muséographie sont-elles complémentaires ?}

Les musées de société consacrés aux faits migratoires ou aux diasporas réaffirment dans un contexte de montée des mouvements xénophobes combien les migrations sont partie intégrante des histoires de chaque pays. Aussi, dans le cadre de ce numéro anniversaire de la REMI, il nous a paru intéressant " d'observer " quelques muséums de renommée internationale (à Paris, Hambourg, Berlin, Tel-Aviv et Moscou) consacrés à l'histoire des migrations et des sociétés juives en diaspora, à un moment où les connaissances, en termes de narrations et de méthodologies conceptuelles, étaient suffisamment " opérantes " pour exposer les faits dans leur complexité. Les savoirs scientifiques ont-ils suffisamment pu peser sur les représentations muséographiques ? Quelles sont les " conventions d'écriture "lorsque les contraintes narratives des directeurs et conservateurs de musées vis-à-vis de leurs tutelles peuvent être en opposition avec les exigences des chercheurs et lorsque les muséographes ont, par souci de vulgarisation, tendance à vouloir privilégier une approche narrative et linéaire des migrations ?

\section{Migrations in Museums! Are Social Sciences and Museography Complementary?}

Museums related to Migrations or Diasporas, in a context of rising xenophobia, reinforce the idea how migrations are full part of National Histories. As part of this project of anniversary volume of REMI, we thought it would be interesting to analyze the exhibitions of few museums internationally reputed (in Paris, Hamburg, Berlin, Tel-Aviv and Moscow) dedicated to Migrations and Jewish Societies in Diasporas at the time when scientific knowledge in terms of narration or conceptual approach sufficiently operational to expose the facts in their complexity. Is scientific knowledge strong enough facing museum projects? Where can the attention to detail go in facing narrative models promoted by director and curator museums, and their trustees, or when historians are contend with museographers who are privileging narrative and linear approaches of migrations?

\section{¡La migración en los museos! ¿Son complementarias las ciencias sociales y la museografía?}

En un contexto en el que los movimientos xenófobos están en auge, los museos dedicados a los fenómenos migratorios o a las diásporas son una muestra de hasta qué punto las migraciones forman parte de la historia de cada país. Con motivo del aniversario de la REMI, nos parece interesante "observar de cerca» varios museos de prestigio internacional (de París, Hamburgo, Berlín, Tel-Aviv y Moscú) que se centran en la historia de las migraciones y en las sociedades judías de la diáspora en una época en la que los aportes científicos, en términos de narración y de metodologías conceptuales, son lo suficientemente "operantes» para mostrar los hechos en toda su complejidad. ¿Han podido tener los saberes científicos el peso suficiente sobre las representaciones museográficas? ¿Qué «normas de escritura» aplican los directores y los comisarios de los museos cuando sus responsabilidades narrativas se oponen a las exigencias de los investigadores? ¿Y cuándo, debido a la vulgarización, los museógrafos se ven obligados a querer privilegiar una perspectiva narrativa y lineal de las migraciones? 